\title{
Research Square \\ Course of long COVID symptoms over time in the ComPaRe long COVID prospective e-cohort
}

\author{
Viet-Thi Tran ( $\nabla$ thi.tran-viet@aphp.fr) \\ Paris University https://orcid.org/0000-0003-1863-6739 \\ Raphael Porcher \\ Paris University \\ Isabelle Pane \\ Assistance Publique Hôpitaux de Paris \\ Philippe Ravaud \\ Paris Descartes University
}

\section{Article}

Keywords: prospective cohort, post-COVID-19 disease, long COVID, patient-reported outcome measures, quality of life

Posted Date: November 17th, 2021

DOI: https://doi.org/10.21203/rs.3.rs-753615/v2

License: (1) (1) This work is licensed under a Creative Commons Attribution 4.0 International License. Read Full License

Version of Record: A version of this preprint was published at Nature Communications on April 5th, 2022. See the published version at https://doi.org/10.1038/s41467-022-29513-z. 


\section{Abstract}

About $10 \%$ of people infected by severe acute respiratory syndrome coronavirus 2 experience post COVID19 disease. We analysed data from 968 adult patients (5350 person-months) with a confirmed infection enrolled in the ComPaRe long COVID cohort, a disease prevalent prospective e-cohort of such patients in France. Day-by-day prevalence of post COVID-19 symptoms was determined from patients' responses to the Long COVID Symptom Tool, an online validated self-reported questionnaire assessing 53 post COVID19 disease symptoms. One year after symptom onset, $84.9 \%$ patients still reported their persistence, with a progressively lower prevalence of $27 / 53$ symptoms (e.g., loss of taste/smell); $18 / 53$ symptoms (e.g., dyspnoea) were stable, while the prevalence of $8 / 53$ symptoms (e.g., paraesthesia) had increased. The disease impact on patients' lives began increasing 6 months after onset, as patients realized they had a chronic disease. Our results should be useful for researchers seeking the potential pathophysiological mechanisms underlying post COVID-19 disease.

\section{Background}

As of October 2021, about 200 million people worldwide had been infected by the severe acute respiratory syndrome coronavirus 2 (SARS-CoV-2), the pathogen responsible for coronavirus disease 2019 (COVID19) ${ }^{1}$. According to the United Kingdom Office for National Statistics, about $10 \%$ of them will experience post COVID-19 disease or "long COVID", that is, the persistence of symptoms such as fatigue, dyspnoea, chest pain, cognitive disturbances, or arthralgia, for several weeks to months after their initial SARS-CoV-2 infection ${ }^{2}$. Research has mainly focused on the occurrence of specific long-term complications among hospitalised and non-hospitalised patients recruited during their acute COVID-19 infection ${ }^{3-7}$. To our knowledge, only a handful of studies have investigated the longitudinal evolution of symptoms among patients with persisting symptoms (i.e., with post COVID-19), but they have generally been small, either retrospective or limited to single centres or both ${ }^{8,9}$. In this study, we used data from a large nationwide cohort of patients to reconstruct the day-by-day course of their symptoms from onset to 1 year after the acute phase of the infection.

\section{Results}

The ComPaRe Long COVID cohort is an ongoing nationwide e-cohort of patients with post COVID-19 disease in France, nested in the ComPaRe research programme (www.compare.aphp.fr), an umbrella ecohort of patients with chronic conditions ${ }^{10}$. The cohort started in December 2020, and recruitment is ongoing. Among the 1859 patients included in the ComPaRe Long COVID cohort on October 19, 2021, we analysed the data from the 968 patients reporting: 1) a laboratory-confirmed COVID-19 infection with a positive test for SARS-CoV2 by PCR swab and/or serologic assay; 2 ) symptoms persisting for at least 2 months after their onset; and 3) enrolment in the cohort by August 1,2021, and thus at least 2 months of follow-up in this analysis (Supplementary material 1). To improve the representativeness of results, we weighted observations by calibration on margins, so that the weighted distribution of age $(<24,25-34,35-$ 
49,5 $0-69$, and $\geq 70$ years), gender, and hospitalisation during the acute phase of the disease (Yes/No) match the data from the UK Office of National Statistics (2 September 2021 data) ${ }^{11,12}$. Raw and weighted patients' characteristics are presented in Supplementary material 2 . Hereafter, results presented always come from the weighted dataset. Raw results are available in the Supplementary materials.

In the weighted data, patients' median age was 48 years (interquartile range 32 to 56 ) with $57.7 \%$ (559/968) men. In all, 35.1\% (340/968) reported comorbidities, 6.3\% (61/968) had chronic lung diseases, and $4.2 \%(41 / 968)$ high blood pressure. The median time between symptom onset and the last follow-up was 174 days (IQR 97 to 284 days). Among participants, 75 (7.7\%) had been hospitalised during their acute disease and 34 (3.5\%) had been admitted to an intensive care unit (ICU).

Participants were followed up every 60 days with online questionnaires available on computer or smartphone. At each observation point, patients were first asked if they still had symptoms related to COVID-19. Those reporting the persistence of symptoms completed the long COVID symptom tool (ST) and impact tool (IT), a pair of validated patient-reported instruments assessing respectively 53 symptoms and 6 dimensions of patients' lives that can be affected by the disease ${ }^{13}$. Those reporting that they no longer had any symptoms were asked to report the date when they first noticed the absence of symptoms (Online Methods). Overall, our data covered 5350 person-months, with a median follow-up since cohort enrollment of 181 days (interquartile range 118 to 240 days). The proportion of patients lost to follow-up was $<20 \%$ at every follow-up (Supplementary material 3 ).

Remission of symptoms (i.e., disappearance of all symptoms) was observed during follow-up for 150 patients. Among those who reported full symptom remission, 50/150 (33.3\%) subsequently described a relapse for at least one symptom. At 12 months, the probability of symptom persistence (including patients in remission who relapsed) was $84.9 \%$ (95\% Cl 79.8\% to $90.4 \%$ ) (Figure 1).

We used multistate models taking both left and right censoring into account to estimate the longitudinal evolution of symptoms; each patient contributed to the data during his/her follow-up period. Because our cohort included patients enrolled at different times after their initial infection, we were able to reconstruct the prevalence of each symptom, day by day, in the study population (Figure 2a, Supplementary material 4). First, 27 (51\%) symptoms showed a progressive decrease in prevalence over time. Among them, loss of appetite, change/loss of taste, and a cough changed most (>20\% decrease). Second, 18 (34\%) symptoms showed no specific change in prevalence over time. Among them, word finding problems and dyspnoea were the most prevalent; at 360 days, they affected respectively $48 \%$ and $44.5 \%$ of the population. Finally, 8 symptoms showed an increase in prevalence over time. Among them, neck, back, and low back pain and paraesthesia changed most markedly (>10\% increase) (Supplementary material 5). The evolution of symptoms over time in subgroups by age, sex, and presence of comorbidities is presented in Supplementary materials 6, 7, and 8.

Post COVID-19 disease is a relapsing-remitting disease. Sixty days after symptom onset, most of patients reported permanent, daily or weekly symptoms. Over time, relapses became less frequent, with a decrease 
in the proportion of patients reporting weekly or more frequent symptoms (including those reporting permanent symptoms) and a parallel increase in the proportion reporting relapses less than weekly (Supplementary material 9).

Figure $\mathbf{2 b}$ illustrates patients' perceptions of the impact of the disease on their lives, measured by the long COVID IT. In particular, we present the proportion of patients reporting an unacceptable disease state, defined as a score on the long COVID IT above the value at which $>75 \%$ of patients consider that they could not cope with a similar level of lifelong symptoms ${ }^{13}$. This analysis revealed two distinct phases of the disease. In a first phase, from 60 to 180 days, the burden of disease progressively decreased as several symptoms disappeared, while the number of patients reporting an unacceptable symptom state rose slowly to $50 \%$. After 6 months, the proportion of patients reporting an unacceptable disease state increased more rapidly, with $60-70 \%$ of patients considering their disease unacceptable at that time. This secondary increase may correspond to patients' realisation that they had a chronic disease. This Ushaped trend in the evolution of the perception of the impact of the disease on patients' lives over time was found in subgroups defined by age and sex; this finding highlights the disease's important impact on all patients (Supplementary material 10 and 11).

\section{Discussion}

This is the first study to report how the symptoms and impact of post COVID-19 evolve after the acute phase of the disease, in a large prospective cohort of patients with a laboratory-confirmed infection. Among patients with post COVID-19 disease, 85\% still reported symptoms one year after symptom onset. This finding is consistent with observations in a single-centre study in Germany, where $20 \%$ of patients were free from all 14 symptoms under study at 12 months $^{8}$.

The course of symptoms over time highlighted three distinct patterns that offer insight into the aetiologies and mechanisms underlying this disease. First, we observed a decrease in prevalence over time for symptoms such as loss of taste or smell, coughing, or diarrhoea. For example, the prevalence of coughing decreased from $50 \%$ to $20 \%$ of participants within the first 6 months after symptom onset before reaching a plateau. This was similar for loss of smell, with a plateau reached after 8 months. This evolution, indicating recovery from the acute phase, is slower than expected; indeed, most guidelines still consider a cut-off of 12 weeks to distinguish ongoing symptomatic COVID-19 (i.e., signs and symptoms of COVID-19 from 4 to 12 weeks) from post-COVID-19 syndrome. ${ }^{14}$ For other symptoms, we showed that their prevalence increased over time. For example, the prevalence of hair loss increased over time with $8 \%$ and $15 \%$ of participants reporting it at 2 months and one year after onset respectively. Late symptom appearance, especially alopecia, has been reported in other studies and should be further investigated. ${ }^{15}$ Finally, symptoms showing no change of prevalence over time may be caused by mechanisms that do not change rapidly over time, such as deconditioning or post-traumatic stress disorder, or due to a mixture of recovery from acute disease and late-onset symptoms appearing as a consequence of COVID-19 ${ }^{16}$. 
We found some differences in the evolution of symptoms between men and women; and in age groups. We hypothesise that differences may be due either to different underlying causes of the persistent symptoms or to specific factors directly affecting symptoms. For example, several studies have highlighted differences between the sexes in immune responses (in terms of levels of innate immune cytokines such as IL-8 and IL-18, induction of non-classical monocytes, and T cell activation) ${ }^{17}$ and in response to a traumatic event ${ }^{18}$.

Our results demonstrate the substantial impact of post COVID-19 disease on patients' lives. Specifically, the course of patients' perceptions of the impact of the disease changed similarly over time across all subgroups, with an aggravation six months after onset. We hypothesise that this corresponds to patients' realisation that this novel and previously unknown disease is chronic rather than acute. This trend seemed more pronounced among younger patients, for whom post COVID-19 is more likely to be both their first contact with a chronic disease and a biographical disruption of their lives ${ }^{19}$.

Strengths of this study lie in the prospective follow-up of a large population of patients with a confirmed COVID-19 infection and their regular assessment of their symptoms using validated patient-reported outcome measures, developed from patients' lived experience, with excellent reproducibility (intraclass correlation coefficient $0.83,95 \%$ confidence interval 0.80 to 0.86$)^{13}$. The use of online questionnaires reduced social desirability bias and may provide better estimates of the prevalence of sensitive symptoms such as genitourinary or cognitive disorders ${ }^{20}$.

This study has several limitations. The recruitment of volunteers in the ComPaRe long COVID cohort may have selected patients who had overall more symptoms, and our recruitment strategy included a social media and general media campaign that may have selected younger and better educated patients. Second, our sample included a majority of women. Persistent symptoms after COVID-19 are more frequent among women; for example, the odds of persistent fatigue at one year among women compared with men was 1.43 [1.04-1.96] $]^{21}$. To minimise these issues, we used a weighted dataset with weights derived from the September 2021 data from the Office of National Statistics in the United Kingdom ${ }^{11}$. Yet, because our original dataset included fewer men, results in this subgroup may lack precision. More generally, the subgroup analyses were post-hoc, and should be considered as exploratory. Third, in this study we included patients with a history of confirmed SARS-CoV-2 infection who had symptoms for at least two months, in accordance with the WHO's recent consensus definition of post COVID-19 disease ${ }^{22}$. However, our data prevented us from ascertaining whether an alternative diagnosis might explain the symptoms. Fourth, as patients could enrol in the cohort at any time point after their initial infection (with an interval between symptom onset and enrolment exceeding 300 days for $25 \%$ of participants), we chose to minimise memory bias by not asking retrospectively for symptoms experienced during the infection's acute phase. This limits our ability to describe how symptoms change immediately after the acute phase of the disease. Finally, in view of the limited number of patients who were hospitalised in ICUs in our study, our results cannot be generalised to this specific population. 
In conclusion, our study shows that most patients with post COVID-19 disease have symptoms evolving in different patterns but persisting through one year. Recovery from the acute infection is a slow process, and the prevalence for most symptoms decreased over time before plateauing six to eight months after onset. Our results should be useful for researchers seeking the potential pathophysiological mechanisms underlying post COVID-19 disease and should also help physicians to inform their patients about the potential course of this disease.

\section{Declarations}

Acknowledgements

The authors thank Elise Diard, Carolina Riveros and Clara Marre for their work in the ComPaRe e-cohort and Jo Ann Cahn for editing

Contributions

Generated the idea: VTT, Conceived and designed the experiments: VTT, RP and PR, Collected data: VTT, IP and PR. Analysed data: VTT and RP; Wrote the first draft of the manuscript: VTT, Contributed to the writing of the manuscript: VTT, RP, IP and PR; ICMJE criteria for authorship read and met: VTT, IP, RP and PR. Agree with manuscript results and conclusions: VTT, RP, IP and PR. VTT is the guarantor, had full access to the data in the study, and takes responsibility for the integrity of the data and the accuracy of the data analysis.

Competing interests

The authors declare no competing interests and no financial associations that may be relevant or seen as relevant to the submitted manuscript. The authors have no association with commercial entities that could be viewed as having an interest in the general area of the submitted manuscript.

\section{References}

1. World Health Organization. WHO Coronavirus Disease (COVID-19) Dashboard. Vol. 2020 (World Health organization, 2020).

2. Carfì, A., Bernabei, R. \& Landi, F. Persistent Symptoms in Patients After Acute COVID-19. Jama 324, 603-605 (2020).

3. Huang, C., et al. 6-month consequences of COVID-19 in patients discharged from hospital: a cohort study. Lancet (London, England) 397, 220-232 (2021).

4. Nehme, M., et al. COVID-19 Symptoms: Longitudinal Evolution and Persistence in Outpatient Settings. Annals of internal medicine (2020). 
5. Ayoubkhani, D., et al. Post-covid syndrome in individuals admitted to hospital with covid-19: retrospective cohort study. BMJ (Clinical research ed.) 372, n693 (2021).

6. Nehme, M., Braillard, O., Chappuis, F., Courvoisier, D.S. \& Guessous, I. Prevalence of Symptoms More Than Seven Months After Diagnosis of Symptomatic COVID-19 in an Outpatient Setting. Annals of internal medicine (2021).

7. Taquet, M., Geddes, J.R., Husain, M., Luciano, S. \& Harrison, P.J. 6-month neurological and psychiatric outcomes in 236379 survivors of COVID-19: a retrospective cohort study using electronic health records. The lancet. Psychiatry (2021).

8. Seeßle, J., et al. Persistent symptoms in adult patients one year after COVID-19: a prospective cohort study. Clinical infectious diseases : an official publication of the Infectious Diseases Society of America (2021).

9. Davis, H., et al. Characterizing Long COVID in an International Cohort: 7 Months of Symptoms and Their Impact. medRxiv (2020).

10. Tran, V.T. \& Ravaud, P. COllaborative open platform E-cohorts for research acceleration in trials and epidemiology. Journal of clinical epidemiology 124, 139-148 (2020).

11. Office for National Statistics. Prevalence of ongoing symptoms following coronavirus (COVID-19) infection in the UK: 2 September 2021. (London, UK, 2021).

12. Institut National de la Statistique et des etudes économiques. La macro SAS CALMAR. in Modèles, méthodes et outils statistiques, Vol. 2019 (Institut National de la Statistique et des etudes économiques, 2018).

13. Tran, V.T., et al. Development and validation of the long covid symptom and impact tools, a set of patient-reported instruments constructed from patients' lived experience. Clinical infectious diseases : an official publication of the Infectious Diseases Society of America (2021).

14. NICE. COVID-19 rapid guideline: managing the long-term effects of COVID-19. (NICE, 2020).

15. Miyazato, Y., et al. Prolonged and Late-Onset Symptoms of Coronavirus Disease 2019. Open forum infectious diseases 7, ofaa507 (2020).

16. Nalbandian, A., et al. Post-acute COVID-19 syndrome. Nature medicine 27, 601-615 (2021).

17. Takahashi, T., et al. Sex differences in immune responses that underlie COVID-19 disease outcomes. Nature 588, 315-320 (2020).

18. Christiansen, D.M. \& Berke, E.T. Gender- and Sex-Based Contributors to Sex Differences in PTSD. Current psychiatry reports 22, 19 (2020). 
19. Bury, M. Chronic illness as biographical disruption. Sociology of health \& illness 4, 167-182 (1982).

20. van Gelder, M.M., Bretveld, R.W. \& Roeleveld, N. Web-based questionnaires: the future in epidemiology? American journal of epidemiology 172, 1292-1298 (2010).

21. Huang, L., et al. 1-year outcomes in hospital survivors with COVID-19: a longitudinal cohort study. Lancet (London, England) 398, 747-758 (2021).

22. Soriano, J., et al. A clinical case definition of post COVID-19 condition by a Delphi consensus. (World Health Organization, 2021).

\section{Figures}

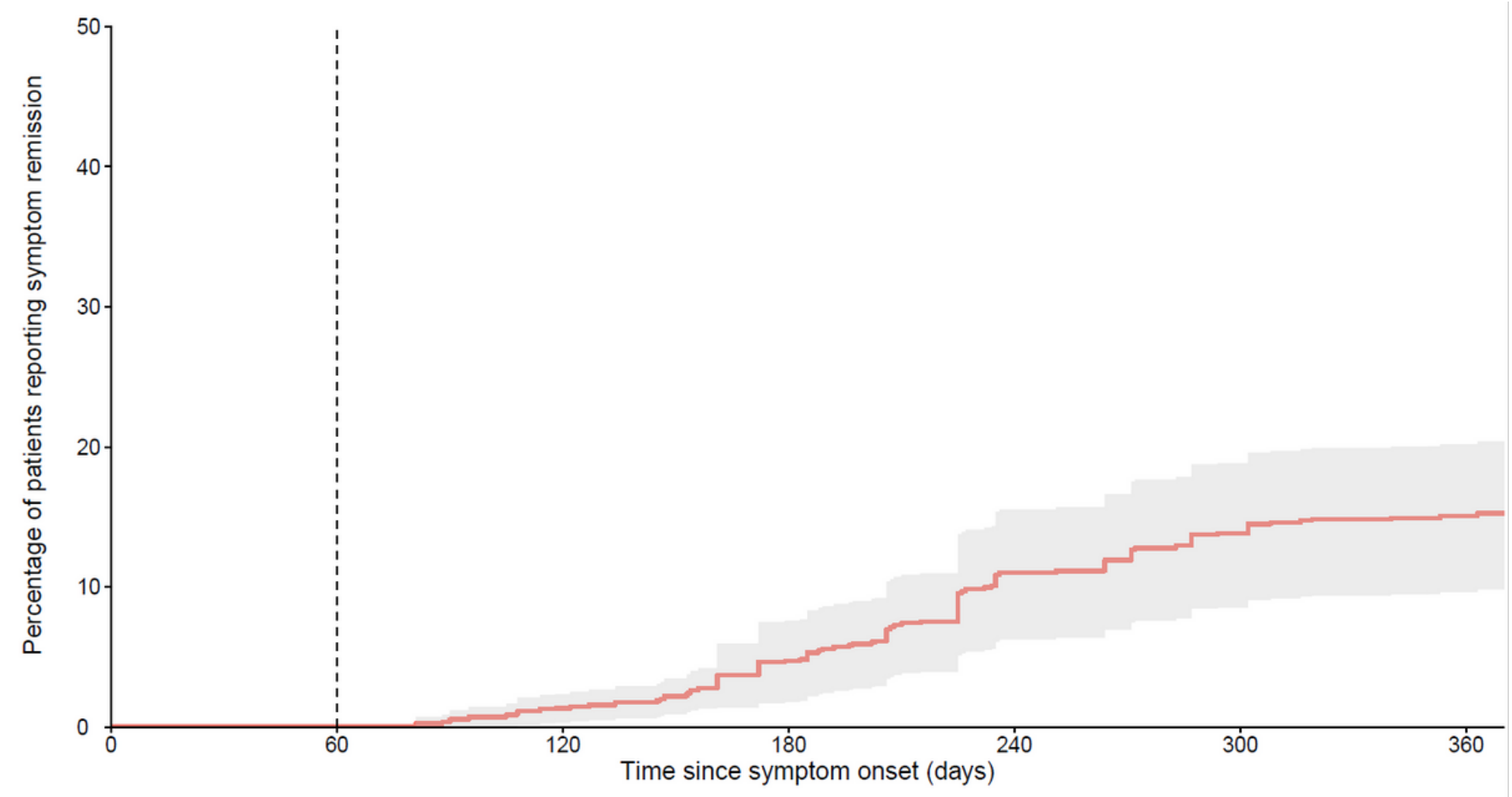

Number at risk

146

262

371

357

424

313

\section{Figure 1}

Cumulative event curve for remission of post COVID-19 symptoms. Time of remission was defined as the first time that patients reported no longer experiencing any symptoms of post COVID-19 disease. The time at risk started at entry in the cohort and ended on October 10, 2021. Follow-up data were censored at the participants' latest observation point. 

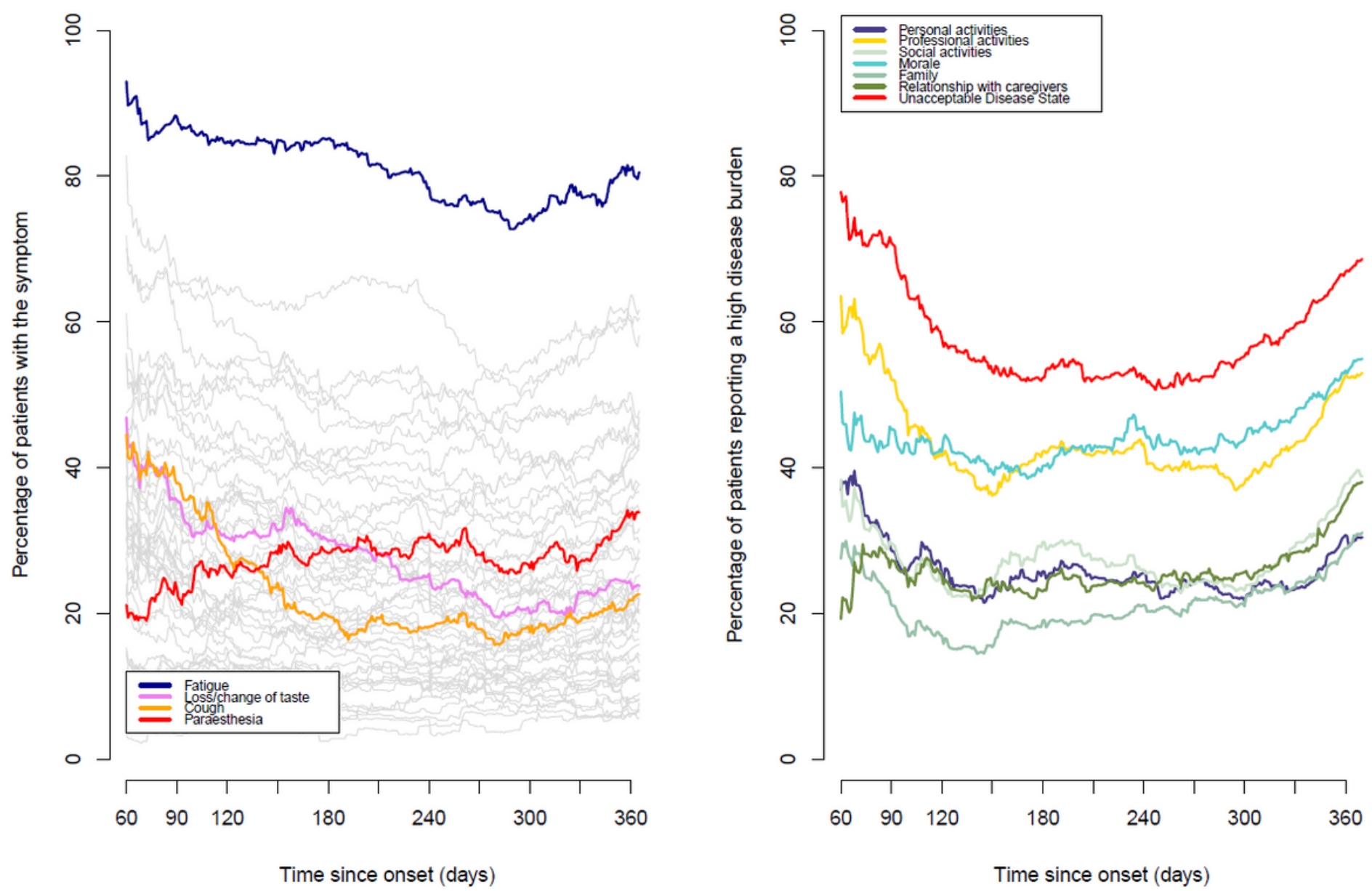

\section{Figure 2}

Day-by-day trends in the prevalence of post COVID-19 disease symptoms (A) and of their impact on patients' lives (B). A: The figure presents the day-by-day prevalence of each of the 53 symptoms assessed by the Long COVID ST (grey lines). Examples of specific symptoms have been highlighted (coloured lines). For each symptom and at each observation point, we assumed that patients could either be "experiencing" or "not experiencing" the symptom. We assumed that their state at an arbitrary time was the same as the state at their previous observation point and that their states before their first observation and after their last observation are unknown. B: The figure presents the day-by-day evolution of the 6 domains of patients' lives that can be affected by post COVID-19 disease and are assessed by the Long COVID IT. For each item and at each observation point, we modelled patients answers as either "reporting" a significant impact of the disease on this domain" (i.e., item score $>7$ ) or "not reporting" this impact (i.e., item score $<8)$. We assumed that their state at an arbitrary time was the same as the state at their previous observation point and that their states before their first observation and after their last observation are unknown. The red lines represent a similar model for the Patient Acceptable Symptomatic State (PASS) of the long COVID IT, which is the long COVID IT score below which $75 \%$ of patients find that their disease state is acceptable. 


\section{Supplementary Files}

This is a list of supplementary files associated with this preprint. Click to download.

- OnlinemethodsREV.docx

- SupplementarymatREV.docx 